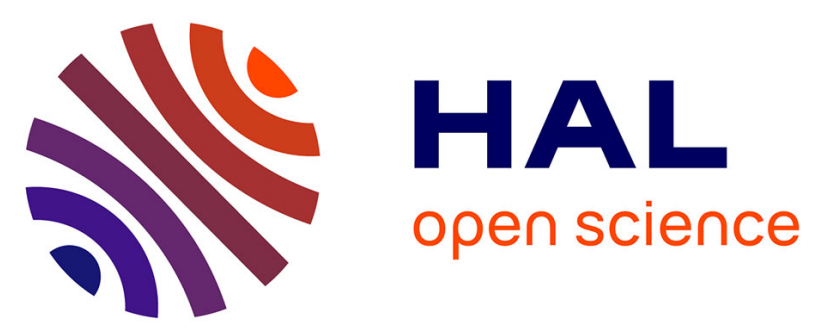

\title{
Improved and isotropic resolution in tomographic diffractive microscopy combining sample and illumination rotation
}

Stanislas Vertu, Jens Flügge, Jean-Jacques Delaunay, Olivier Haeberlé

\section{To cite this version:}

Stanislas Vertu, Jens Flügge, Jean-Jacques Delaunay, Olivier Haeberlé. Improved and isotropic resolution in tomographic diffractive microscopy combining sample and illumination rotation. Central European Journal of Physics, 2011, 9 (4), pp.969-974. 10.2478/s11534-011-0018-3 . hal-00851880

\author{
HAL Id: hal-00851880 \\ https://hal.science/hal-00851880
}

Submitted on 19 Aug 2013

HAL is a multi-disciplinary open access archive for the deposit and dissemination of scientific research documents, whether they are published or not. The documents may come from teaching and research institutions in France or abroad, or from public or private research centers.
L'archive ouverte pluridisciplinaire HAL, est destinée au dépôt et à la diffusion de documents scientifiques de niveau recherche, publiés ou non, émanant des établissements d'enseignement et de recherche français ou étrangers, des laboratoires publics ou privés. 


\section{Editorial Manager(tm) for Central European Journal of Physics}

Manuscript Draft

Manuscript Number: CEJP-D-10-00145R1

Title: Improved and isotropic resolution in tomographic diffractive microscopy combining sample and illumination rotation

Article Type: Research Article

Section/Category: Optics and Lasers

Keywords: Tomography; Holography; Fourier Optics; Image Reconstruction

Corresponding Author: Olivier HAEBERLE, Ph.D

Corresponding Author's Institution:

First Author: Stanislas Vertu

Order of Authors: Stanislas Vertu;Jens Flügge;Jean-Jacques Delaunay;Olivier HAEBERLE, Ph.D

Abstract: Tomographic Diffractive Microscopy is a technique, which permits to image transparent living specimens in three dimensions without staining. It is commonly implemented in two configurations, by either rotating the sample illumination keeping the specimen fixed, or by rotating the sample using a fixed illumination. Under the first-order Born approximation, the volume of the frequency domain that can be mapped with the rotating illumination method has the shape of a "doughnut", which exhibits a so-called "missing cone" of non captured frequencies, responsible for the strong resolution anisotropy characteristic of transmission microscopes. When rotating the sample, the resolution is almost isotropic, but the set of captured frequencies still exhibits a missing part, the shape of which resembles that of an apple core. Furthermore, its maximal extension is reduced compared to tomography with rotating illumination. We propose various configurations for tomographic diffractive microscopy, which combine both approaches, and aim at obtaining a high and isotropic resolution. We illustrate with simulations the expected imaging performances of these configurations.

\section{Suggested Reviewers:}

Opposed Reviewers:

Response to Reviewers: Please, see attached document, which contains a letter for the editor, and our answer to the referees 


\title{
Improved and isotropic resolution in tomographic diffractive microscopy combining sample and illumination rotation
}

\author{
Stanislas Vertu†, Jens Flügge†, Jean-Jacques Delaunaył*, Olivier Haeberlé** \\ $\dagger$ Physikalisch-Technische Bundesanstalt (PTB), Bundesallee 100, 38116 Braunschweig, Germany \\ $\ddagger$ Department of Mechanical Engineering, School of Engineering, The University \\ of Tokyo, 7-3-1 Hongo Bunkyo-ku, Tokyo 113-8656, Japan \\ ${ }^{\circ}$ Laboratory MIPS EA2332 - University of Haute Alsace, IUT Mulhouse, 61 rue \\ Albert Camus, 68093 Mulhouse Cedex, France \\ *E-mail: jean@mech.t.u-tokyo.ac.jp, olivier.haeberle@uha.fr
}

(dated: November 8, 2010)

\begin{abstract}
Tomographic Diffractive Microscopy is a technique, which permits to image transparent living specimens in three dimensions without staining. It is commonly implemented in two configurations, by either rotating the sample illumination keeping the specimen fixed, or by rotating the sample using a fixed illumination. Under the first-order Born approximation, the volume of the frequency domain that can be mapped with the rotating illumination method has the shape of a "doughnut", which exhibits a so-called "missing cone" of non-captured frequencies, responsible for the strong resolution anisotropy characteristic of transmission microscopes. When rotating the sample, the resolution is almost isotropic, but the set of captured frequencies still exhibits a missing part, the shape of which resembles that of an apple core. Furthermore, its maximal extension is reduced compared to tomography with rotating illumination. We propose various configurations for tomographic diffractive microscopy, which combine both approaches, and aim at obtaining a high and isotropic resolution. We illustrate with simulations the expected imaging performances of these configurations.
\end{abstract}

Keywords: Tomography, Holography, Fourier Optics, Image Reconstruction.

\section{Introduction}

Conventional transmission microscopes suffer from a poor resolution, especially along the optical axis, because of an incomplete mapping of the observed object frequencies, and deliver intensity-only images, which contrast is linked in a complex manner to the index of refraction distribution of the observed specimen. Tomographic microscopy $[1,2]$ has therefore regain interest in recent years because it allows for the observation of unprepared transparent or quasi-transparent samples, which, due to their low contrast, pose a real challenge in conventional transmission microscopy, and delivers quantitative 3-D images of the specimen in both index of refraction and absorbtion, quantities, which are not or hardly measurable with conventional microscopy.

When neglecting diffraction, the reconstruction of the variations in refractive index of transparent samples is usually obtained by backprojecting the measured sample phase using the Radon transform $[1,2,3,4]$. By applying backprojection, the obtained spatial resolution is isotropic and no information is missing along the rotation axis. However this reconstruction by backprojection can induce artefacts $[4,5]$, and the achievable resolution is limited to about the wavelength of observation.

In order to obtain a better view of the observed sample, high numerical aperture (NA) set-ups should be used in combination with reconstruction techniques, which do not neglect diffraction, usually based on the diffraction tomography theorem $[6,7,8,9]$. In that case, one will speak of Tomographic Diffractive Microscopy (TDM) $[10,11,12,13]$.

The resolution, especially along the optical axis, is better than with holographic microscopy [14], and may approach or even exceed that of confocal microscopy $[15,16,17]$. However, for transmission or reflection tomographic set-ups with fixed specimen, it is always anisotropic. With a rotating specimen, the resolution is almost isotropic [18, 19], but is lower than what is achievable with tomographic diffractive microscopy with illumination rotation.

In this work, we study several configurations, combining sample rotation with illumination rotation and aiming at a final 3-D resolution that is both improved (compared with TDM with sample rotation) and isotropic (contrary to systems with a fixed sample). Simulations are used to estimate the imaging performances, which should be achievable with these improved configurations. 


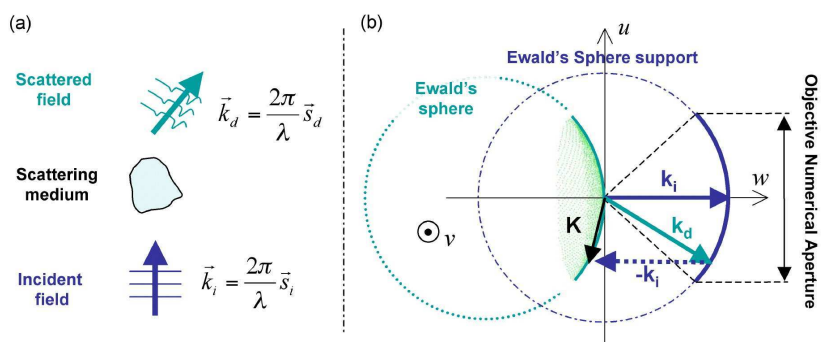

Figure 1: : (a) Definition of the propagation vector of the scattered light from a scattering medium. (b) The Fourier transform of the object scattering potential is distributed over the surface of a spherical cap, the top of which coincides with the frequency origin. Due to the limited numerical aperture of the detection objective, only a cap of this sphere is captured in practice.

\section{TDM with illumination rota- tion}

We first briefly recall the record of object frequencies in holographic microscopy, as this technique is at the heart of all tomographic configurations. For a more detailed description, we refer the interested reader to Refs. $[6,7,8,9]$ for an introduction to the basics of diffraction, and to Refs. [20, 21], for a more detailed description of holographic and tomographic microscopy in the framework of the first order Born approximation.

Figure 1 describes the principle of holographic diffractive microscopy in a transmission set-up, in twodimensions for the sake of simplicity, and in the $(u, w)$ -plane. Here $(u, v, w)$ correspond to Fourier frequencies along the $(\mathrm{x}, \mathrm{y}, \mathrm{z})$ directions, respectively. When illuminated by a plane wave, the observed object scatters waves in all directions (Figure 1(a)), which wavevector extremities depict in the Fourier space the so-called Ewald sphere (Figure 1(b)). Because of the limited numerical aperture of the detection system, only a cap of this sphere can be recorded. In the framework of the first Born approximation (elastic single scattering), the momentum conservation permits to replace the corresponding object waves by a simple translation (in green on Fig. 1(b)).

The lateral $((u, v)$-plane) extension of the Optical Transfer Function (OTF) of holographic microscopy is non negligible. This explains the good lateral resolution of holographic microscopy [14]. However, the longitudinal ( $w$-axis) extension is very small, inducing bad discriminating capabilities of holographic microscopy along the optical axis [14]. The aim of all TDM set-ups is to increase this set of recorded frequencies in order to improve the lateral and/or longitudinal resolution.

For tomographic diffractive microscopy with illumination rotation $[11,12,14]$, one usually uses a condenser to control the incidence of the illumination, with same NA as the objective used for detection (other configurations have been described in [22]). For a full scanning of the condenser aperture, the OTF of the system takes the "doughnut" shape described by Fig. 2. A detailed explanation of its construction can be found
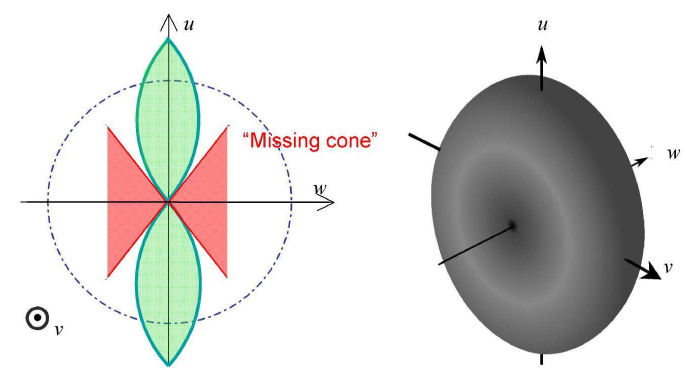

Figure 2: Representation of the optical transfer function of a tomographic diffractive microscope with illumination rotation. While the resolution is doubled with respect to a conventional transmission microscope, the frequency support exhibits a missing-cone (in red) of uncaptured frequencies, responsible for the poorer resolution along the optical axis.

in $[12,20]$. The lateral extension is twice that of holographic microscopy [12], but the most noticeable feature is the now non-negligible extension along the optical axis, which permit a better discrimination along the optical axis [14]. The frequency support is identical to that of conventional transmission microscopy. However, in conventional microscopy, high frequencies are strongly attenuated. In tomographic diffractive microscopy, thanks to the use of coherent detection, the high frequencies are recorded without attenuation. As a consequence, the resolution is improved by a factor 2, as explained in Refs. [12, 20, 21] and experimentally demonstrated in Ref. [15]. As in any transmission set-up, the OTF however presents a so-called missing cone (in red) of unregistered frequencies, responsible for a much lower resolution along the optical axis than in the transverse plane.

A variant of this set-up has been proposed, with a oneaxis illumination rotation only [2]. In that case, the OTF takes a more complex "peanut" shape [23], but this setup has the advantage that very fast scanning speed is achievable, rendering possible the observation of dynamic phenomena within living specimens [24, 25, 26].

\section{TDM with sample rotation}

Because of the strong resolution anisotropy of tomographic diffraction microscopy with illumination rotation, other configurations have been studied [21], among them, tomographic diffraction microscopy with specimen rotation.

In this configuration, the specimen is rotated under the microscope objective. The successive sets of measured object frequencies are combined in Fourier space by rotating back the captured cap of the Ewald sphere (see Fig. 1 ). The resulting optical transfer function is depicted on Fig. 3, and takes the shape of a "ball", but because of the curvature of the successive caps of sphere, a small subset of uncaptured frequencies does remain along the rotation axis (here the $\mathrm{x}$-axis), which takes a characteristic shape of an apple-core [18, 19].

The resolution is therefore almost isotropic, but is 

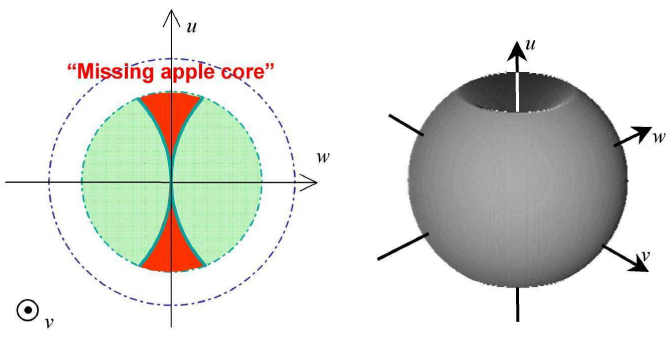

Figure 3: Representation of the optical transfer function of a tomographic diffractive microscope with specimen rotation. The frequency support is almost spherical, but exhibits a set (in red) of uncaptured frequencies, which as the shape of an apple core.

lower than in the previous configuration for two reasons. First, the use of a constant illumination (along the optical axis) limits the set of captured frequencies at each step to a cap of sphere, which summit corresponds to the frequency origin. With TDM with illumination variation, it is indeed possible to extend the set of captured frequencies, because the rotation of the illumination (and not the specimen) translates actually into a shift to higher frequencies of the registered specimen information (see Refs. [12, 20, 21] for a complete description).

Then, rotating the specimen is usually performed by embedding the sample within a micropipette or a capillary $[1,2,5,27,28,29]$, so that it is both easy to handle and to rotate. The specimen itself may be of cylindrical geometry [4]. A very thin syringe needle may also be used to guide the rotation [30]. These sample manipulations require more free space under the microscope objective, which explains that tomographic microscopy with specimen rotation has up-to-now been performed with lower NA objectives, leading to an (isotropic, but) intrisinquely lower resolution that TDM with illumination rotation, which can use the highest NA objectives, the sample being classically prepared within a glass slide and a cover. In some cases, the sample itself may be rotated without the requirement of additional handling hardware [31], but in that case, long working distances, low NA objectives have to be used.

\section{Combined approaches}

We propose to combine TDM with illumination rotation together with a specimen rotation, in order to perform data acquisition, which allows for a simultaneous isotropic and improved resolution. This idea stems from the fact that the missing cone in the first configuration corresponds to the optical axis of the acquisition set-up, while the missing apple-core in the second configuration is oriented along the specimen rotation axis, which itself is usually perpendicular to the optical axis.

Three configurations are studied:

1) Combining the "doughnut" from Fig. 2 with the "ball" of Fig. 3. This requires a precise rotation of the sample with a large number of intermediary steps, typically 200 in TDM [5] and 90 in tomographic microscopy with an inverse Radon reconstruction [1]. The "doughnut" OTF is obtained without sample mouvement by optical tilting only, and therefore, allows for high precision and high speed $[2,24,25,26]$. The final OTF corresponds to the addition of the "ball" OTF (Fig. 4(b)) with the "doughnut" OTF (Fig. 4(c)), and is depicted by Fig. $4(\mathrm{~d})$. No missing cone remains, but the extension of this OTF remains smaller along the optical $w$-axis than in the $(u, v)$ transverse plane.

2) Combining several "doughnuts" with a limited set of specimen rotations around the x-axis. This would have the advantage of strongly decreasing the required number of specimen rotations. Figure 4(e) depicts the final OTF for two acquisitions after a $90^{\circ}$ rotation of the sample. Similarly, Figures $4(\mathrm{f}, \mathrm{g})$ show the final OTF after 4 and 8 rotations of the sample, respectively. Figure 4(h) shows the completely filled sphere for a very large (infinite) number of specimen rotations. Note that already for 8 specimen rotations only is the final OTF (Fig. 4(g)) very close to the ideal case depicted by Fig. 4(h). The resolution in that case would be the same in all directions, and noticeably improved compared to holographic microscopy or conventional transmission microscopy.

3) Combining several "doughnuts" from Fig. 2, with $90^{\circ}$ specimen rotations along both $\mathrm{x}$-axis and y-axis. Figure 4(i) shows for example the OTF obtained with three acquisitions only, after one rotation of the sample along the $\mathrm{x}$-axis and one along the $\mathrm{y}$-axis. The resolution would be the same along the three directions, but the OTF still exhibits regions with uncaptured frequencies. More rotation would be necessary for a more isotropic coverage of the object frequencies. Furthermore, the usual specimen preparation within a glass capillary for rotation prevents a possible rotation along a second axis.

\section{Simulations}

We now study the reconstructions of a synthetic object, mimicking a bacteria, and composed of a cylinder closed at both ends by half spheres, and comprizing two solid spheres, and considering the various proposed set-ups. It is depicted on Fig. 4(a) by (x-y), (x,z) and (y-z) views.

The parameters for the simulations are: $633 \mathrm{~nm}$ illumination wavelength, $\mathrm{NA}=1.2$ water immersion objective. The Abbe resolution is in that case $264 \mathrm{~nm}(\lambda / 2 \mathrm{NA})$, and the tomographic resolution $132 \mathrm{~nm}(\lambda / 4 \mathrm{NA})[12]$. The membrane-like object has a length of $6.5 \mu \mathrm{m}$, a width of $2.77 \mu \mathrm{m}$, a "membrane" thickness of $90 \mathrm{~nm}$, and contains two solid spheres with diameter $830 \mathrm{~nm}$ and 600 $\mathrm{nm}$, respectively. All simulations were performed using Matlab, by filtering the Fourier spectrum of the object, taking into account the specific OTF corresponding to the various proposed configurations.

Figures 4(b') and 4(c') show the simulated image for TDM with sample rotation and for TDM with illumination rotation, respectively. Note that the transverse resolution of the latter is better, but a strong elonga- 
tion, characteristic of transmission microscopes, and due to the missing cone is clearly visible on the $(\mathrm{x}-\mathrm{z})$ view.

Figure 4(d') shows the simulated image for TDM with sample rotation and illumination rotation. Note that no strong elongation along the optical axis is visible anymore (the missing cone of the "doughnut" OTF is filled by the "ball" OTF), but the resolution is not isotropic, because of the OTF not having the same extension along the optical axis than in the transverse plane.

Figures 4(e'-h') show the results obtained for multiangle TDM with illumination rotation, with $2,4,8$ and a very large number of specimen rotations. Figure 4(e') seems to indicate a good reconstruction, but the large gaps still present in the OTF indeed translate into lower image quality, as illustrated by cuts along the x-rotation axis, after a $45^{\circ}$ rotation around this axis (Fig. 4(e"), see arrow). The situation improves with 4 specimen rotations, and there is no noticeable difference between the reconstruction with a very large number of rotations and the reconstruction with 8 rotations, which therefore seems to constitute an excellent compromise between number of acquisitions and reconstruction quality.

Figure 4(i) shows the image simulated for two successive $90^{\circ}$ specimen rotations along the $\mathrm{x}$-axis, then the y-axis. The OTF still presents gaps, indicating that a larger number of rotations would be necessary, as illustrated by Fig. 4(i") (same view as for Fig. 4(e")).

Note the artefacts visible around the objects. They are Gibbs oscillations, characteristic of filtering in Fourier space, and inherent to reconstruction of high contrast specimens under the first Born approximation. Similar artefacts have been observed in experimental images [15]. These kind of artefacts may be simply filtered [32, 33], but, strictly speaking, this comes at the expense of resolution. Other artefacts are the remaining deformations of the observed sample caused by the missing frequencies. In order to further improve the images, a possible approach would be to make use of constraint deconvolution, which as been successfully implemented for holographic [34] and tomographic microscopy with 1D illumination scanning $[24,25,26]$. More elaborate reconstruction algorithms may also be used for highly contrasted specimens $[35,36]$. Note also that, when a priori information can be introduced in the inversion algorithm, the resolution of the reconstruction can be much better than that imposed by the diffraction limit, even for weakly diffracting specimen [37, 38]. These methods however are characterized by a much-increased algorithmic complexity, therefore resulting in computationnaly expensive and time consuming specimen reconstructions.

\section{Conclusion}

Tomographic diffractive microscopy is a promising technique for imaging unlabelled specimens. The two most common approaches, specimen rotation or illumination rotation, are limited in terms of resolution. We have studied numerically several options, which combine these two methods, in order to allow for an optimal specimen reconstruction. The best compromise in terms of experimental simplicity with respect to image quality would be to use TDM with illumination variation, combined to a small number ( 4 or better 8 ) of specimen rotations along one axis, which may be performed mechanically $[1,5,27,28,29,30]$ or optically [39].

Optical tweezers may be used to perform a rotation along one or two axes [40], without mechanical contact with the sample. Similarly, rotation of the sample induced by electric fields may be used [41]. Such devices have not yet, to our knowledge, been used in the framework of optical tomography, but may have several advantages. They permit to handle specimens between a slide and a cover glass, which remains the preferred option for preparing microscopic samples. Doing so will also permit to use high NA objectives (contrary to microcapillaries, requiring a larger working distance, hence a lower NA).

Combining the specimen rotation with illumination rotation permits to simplify the optical system required to capture the entire specimen Fourier spectrum: the twoobjective set-up, similar to 4Pi microscopy [42] and proposed in Ref. [12], necessitates a double interferometer, and three successive acquisitions (one in transmission, and two in reflection mode). Furthermore, even with high NA objectives, missing frequencies still exist [12]. However, no specimen rotation is needed, allowing its use for imaging tissues, or complex structures, and not only individual structures, like isolated cells, pollen grains, or diatomes.

Recently, a novel approach, called mirror assisted tomography [43] has been proposed to simplify the twoobjective detection system of Lauer [12]. This promising technique may also be combined with specimen rotation. In that case, only two acquisitions (with a specimen rotated by $90^{\circ}$ along one axis) would permit to recover the maximum allowed sample Fourier spectrum (Fig. 4(h)), with almost no remaining gap. This mirror assisted tomography (which however has not yet been demonstrated experimentally), would probably constitute the easiest approach for performing isotropic-, ultimate resolution tomographic diffractive microscopy.

\section{References}

[1] F. Charrière, A. Marian, F. Montfort, J. Kuehn, T. Colomb, E. Cuche, P. Marquet, C. Depeursinge, Opt. Lett. 31, 178 (2006)

[2] W. Choi, C. Fang-Yen, K. Badizadegan, S. Oh, N. Lue, R. R. Dasari and M. S. Feld, Nature Methods 4, 717 (2007)

[3] S.Vertu, M. Ochiai, M. Shuzo, I. Yamada, J.-J. Delaunay, O. Haeberlé, Y. Okamoto, European Conference on Biomedical Optics, Munich (Germany), June 15-22 (2007), Proc. SPIE vol 6627, paper 66271A (2007)

[4] W. Gorski, W. Osten, Opt. Lett. 32, 1977 (2007) 
[5] S. Vertu, M. Ochiai, M. Shuzo, I. Yamada, J.-J. Delaunay, O. Haeberlé, Y. Okamoto, Photonics West 2008, San Jose, January 19-24 (2008), Proc. SPIE vol. 6861, paper 686103 (2008)

[6] E. Wolf, Opt. Comm. 1, 153 (1969)

[7] R. Da̋ndliker, K. Weiss, Opt. Comm. 1, 323 (1970)

[8] M. Born, E. Wolf, Principles of Optics, Pergamond Press (1991)

[9] J.D. Jackson, Classical Electrodynamics, Wiley, New York (1999)

[10] S. Kawata, O. Nakamura, S. Minami, J. Opt. Soc. Am. A 4, 292 (1987)

[11] T. Noda, S. Kawata and S. Minami, Appl. Opt. 31, 670 (1992)

[12] V. Lauer, J. Microscopy 205, 165 (2002)

[13] B. Simon, M. Debailleul, V. Georges, V. Lauer and O. Haeberlé, Eur. Phys. J. Appl. Phys. 44, 29 (2008)

[14] M. Debailleul, B. Simon, V. Georges, O. Haeberlé and V. Lauer, Meas. Sci. Technol. 19, 074009 (2008)

[15] M. Debailleul, V. Georges, B. Simon, R. Morin and O. Haeberlé, Opt. Lett. 34, 79 (2009)

[16] B. Simon, M. Debailleul, A. Beghin, Y. Tourneur, and O. Haeberlé, J. Biophotonics 3, 462 (2010)

[17] M. Sarmis, B. Simon, M. Debailleul, B. Colicchio, V. Georges, J.-J. Delaunay and O. Haeberlé, J. Mod. Opt. $57,740(2010)$

[18] S. Vertu, I. Yamada, J.-J. Delaunay, O. Haeberlé, J. Flügge, European Conference on Biomedical Optics, Munich (Germany), June 14-18 (2009) Proc. of SPIE Vol. 7390, paper 73901D (2009)

[19] S. Vertu, J.-J. Delaunay, I. Yamada and O. Haeberlé, Centr. Eur. J. of Phys. 7, 22, (2009)

[20] O. Haeberlé, A. Santenac and H. Giovaninni, An introduction to diffractive tomographic microscopy, in Modern Research and Educational Topics in Microscopy 3, Vol. II Editors: Antonio Mendez Vilas, Jesus Diaz Alvarez, Formatex, 2007

[21] O. Haeberlé, K. Belkebir, H. Giovaninni and A. Santenac, J. Mod. Opt. 57, 686 (2010)

[22] N. Streibl, J. Opt. Soc. Am. A 2, 121 (1985)

[23] S. S. Kou and C. J. R. Sheppard, Opt. Lett. 33, 2362 (2008)

[24] Y. Park, M. Diez-Silva, G. Popescu, G. Lykotrafitis, W. Choi, M. S. Feld, and S. Suresh, PNAS 105, 13730 (2008)

[25] N. Lue, W. Choi, G. Popescu, K. Badizadegan, R. R. Dasari, and M. S. Feld, Opt Exp. 16, 16240 (2008)

[26] Y. Sung, W. Choi, C. Fang-Yen, K. Badizadegan, R. R. Dasari, and M. S. Feld, Opt. Exp. 17, 266 (2009)

[27] M. Fauver, E. J. Seibel, J. R. Rahn, M. G. Meyer, F. W. Patten, T. Neumann and A. C. Nelson, Opt. Exp. 13, 4210-4223 (2005)

[28] F. Charrière, N. Pavillon, T. Colomb, C. Depeursinge, T. J. Heger, E. A. D. Mitchell, P. Marquet, and B. Rappaz, Opt. Exp. 14, 7005 (2006)
[29] I. Bergoënd, N. Pavillon, F. Charrière, and C. Depeursinge, Optical tomography with digital holographic microscopy, 3rd EOS Topical Meeting on Optical Microsystems (O $\left.\mu \mathrm{S}^{\prime} 09\right), 27$ th - 30th September 2009, Capri, Italy, ISBN 978-3-00-024191-8

[30] A. Barty, K.A. Nugent, A. Roberts, D. Paganin, Opt. Comm. 175, 329 (2000)

[31] A. C. Sullivan and R. R. McLeod, Opt. Exp. 15, 14202 (2007)

[32] V. Mico, Z. Zalevski, P. Garcia-Martinez, J. Garcia, J. Opt. Soc. Am. A 23, 3162 (2006)

[33] T. R. Hillman, T. Gutzler, S. A. Alexandrov, and D. D. Sampson, Opt. Exp. 17, 7873 (2009)

[34] Y. Cotte, M. Fatih Toy, N. Pavillon, and C. Depeursinge, Opt. Exp. 18, 19462 (2010)

[35] K. Belkebir, P. Chaumet and A. Sentenac, J. Opt. Soc. Am. A 22, 1889 (2005)

[36] G. Maire, F. Drsek, J. Girard, H. Giovannini, A. Talneau, D. Konan, K. Belkebir, P. C. Chaumet and A. Sentenac, Phys. Rev. Lett. 102, 213905 (2009)

[37] P. M. Van Den Berg and R. E. Kleinman, Gradient methods in inverse acoustic and electromagnetic scattering, in Large-Scale Optimization with Applications, Part I: Optimization in Inverse Problems and Design, L. T. Biegler, et. al. eds. IMA Volumes in Mathematics and its Applications. Vol. 92, Springer-Verlag, New York, 173-194, 1997

[38] P. C. Chaumet, K. Belkebir and A. Sentenac, Phys. Rev. B 69, 245405 (2004)

[39] M. K. Kreysing, T. Kieling, A. Fritsch, C. Dietrich, J. R. Guck, and J. A. Käs, Opt. Exp. 16, 16984 (2008)

[40] R. Dasgupta, S.K. Mohanti, P.K. Gupta, Biotechnol. Lett. 25, 1625 (2003)

[41] B. Le Saux, B. Chalmond, Y. Yu, A. Trouvé, O. Renaud and S.L. Shorte, J. Microsc. 233, 404 (2009)

[42] S.W. Hell and E. H. K. Stelzer, Opt. Comm. 93, 277 (1992)

[43] E. Mudry, P. C. Chaumet, K. Belkebir, G. Maire and A. Sentenac, Opt. Lett. 35, 1857 (2010) 
(a)

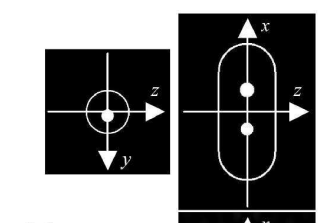

(b)
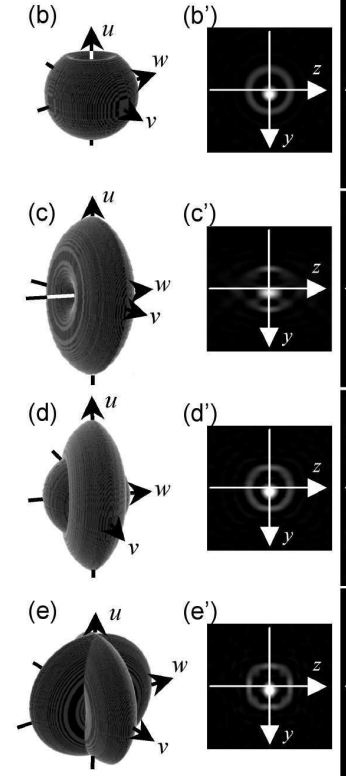

$\left(e^{\prime \prime}\right)$
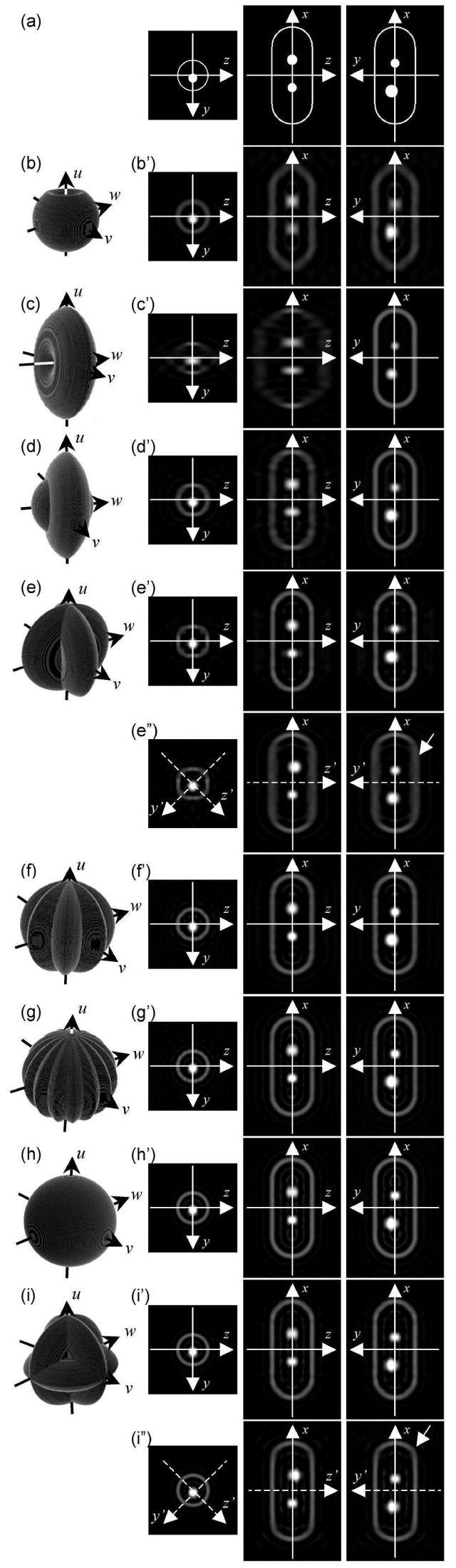

Figure 4: (a): numerical object under consideration viewed as cuts in the $(x-y),(x-z)$, and $(y-z)$ planes. (b)-(i): Optical transfer function of the proposed TDM configurations. (b')-(i'): corresponding simulated images. (e"),(i"): alternate views (see text). 